Europhysics Letters

PREPRINT

\title{
Correlation length of the 1D Hubbard Model at half-filling : equal-time one-particle Green's function
}

\author{
Y.UMEno ${ }^{1}$, M. Shiroishi ${ }^{2}$ and A. KlÜMPER ${ }^{3}$ \\ 1 Department of Physics, Graduate School of Science, University of Tokyo, \\ Hongo 7-3-1, Bunkyo-ku, Tokyo 113, Japan \\ 2 Institute for Solid State Physics, University of Tokyo, Kashiwanoha 5-1-5, \\ Kashiwa, Chiba, 277-8581 Japan \\ 3 Theoretische Physik I, Universität Dortmund, Otto-Hahn-Str. 4, D-44221 Dortmund, \\ Germany
}

PACS. 71.10.Fd - Lattice fermion models (Hubbard model, etc.).

PACS. 71.27.+a - Strongly correlated electron systems; heavy fermions.

PACS. 05.30.Fk - Fermion systems and electron gas.

\begin{abstract}
The asymptotics of the equal-time one-particle Green's function for the half-filled one-dimensional Hubbard model is studied at finite temperature. We calculate its correlation length by evaluating the largest and the second largest eigenvalues of the Quantum Transfer Matrix (QTM). In order to allow for the genuinely fermionic nature of the one-particle Green's function, we employ the fermionic formulation of the QTM based on the fermionic $R$-operator of the Hubbard model. The purely imaginary value of the second largest eigenvalue reflects the $k_{\mathrm{F}}(=\pi / 2)$ oscillations of the one-particle Green's function at half-filling. By solving numerically the Bethe Ansatz equations with Trotter numbers up to $N=10240$, we obtain accurate data for the correlation length at finite temperatures down into the very low temperature region. The correlation length remains finite even at $T=0$ due to the existence of the charge gap. Our numerical data confirm Stafford and Millis' conjecture regarding an analytic expression for the correlation length at $T=0$.
\end{abstract}

The one-dimensional (1D) Hubbard model

$$
\mathcal{H}=-\sum_{j=1}^{L} \sum_{\sigma=\uparrow \downarrow}\left(c_{j+1 \sigma}^{\dagger} c_{j \sigma}+c_{j \sigma}^{\dagger} c_{j+1 \sigma}\right)+U \sum_{j=1}^{L}\left(n_{j \uparrow}-\frac{1}{2}\right)\left(n_{j \downarrow}-\frac{1}{2}\right),
$$

has been intensively studied in condensed matter physics as one of the fundamental models for strongly correlated electrons. In 1968, Lieb and Wu [1] solved the model exactly by means of the coordinate Bethe ansatz method. Based on the Bethe ansatz, many physical quantities have been studied [2-4]. Especially in 1972, Takahashi introduced the string hypothesis and derived the thermodynamic Bethe ansatz equations (TBA) [5], from which several thermal properties were calculated for finite temperature [6-9].

More recently quite a different approach to the thermodynamics of the 1D Hubbard model has been developed based on the quantum transfer matrix (QTM) method [10-30]. In this

(C) EDP Sciences 
approach, the problem is reduced to the eigenvalue problem of the QTM. The free energy and other bulk quantities can be calculated from the largest eigenvalue of the QTM. In 1998, Jüttner, Klümper and Suzuki constructed the QTM utilizing fully the integrability structure of the Hubbard model [25]. For the largest eigenvalue they derived a new type of non-linear integral equations (NLIE). This set of NLIEs turned out to be very powerful especially for the numerical study of the thermal quantities, since it consists of only three auxiliary functions.

One of the remarkable advantages of the QTM approach is that it enables us to study the asymptotics of several correlation functions at finite temperature. Explicit results for the correlation lengths can be derived from the ratios of the next-leading eigenvalues to the largest eigenvalue of the QTM. Using this idea, Tsunetsugu calculated the correlation length of the spin-spin correlations for the half-filled 1D Hubbard model by solving the Bethe Ansatz equations numerically [18]. In the low temperature limit, his results agree with the finite temperature correction of the conformal field theory as $\xi_{\mathrm{s}} \propto v_{\mathrm{s}} / T$. Later this was confirmed analytically by Klümper and Bariev [22].

In this paper, we study another important correlation function of the half-filled 1D Hubbard model, namely the correlation length of the equal-time one-particle Green's function $\left\langle c_{k \sigma}^{\dagger} c_{j \sigma}\right\rangle$. As is well known, the system is an insulator at half-filling and the charge excitation has a gap. There is no effect of the charge gap on the spin-spin correlation function, because the charge excitation is not relevant for the spin-spin correlations. Quite differently, the charge gap plays a significant role for the one-particle Green's function leading to exponential asymptotics even at $T=0$. The quantitative relation, however, between the charge gap and the correlation length is absolutely non-trivial and not well understood yet. In regard to this point, Stafford and Millis [31] conjectured that the correlation length $\xi$ for the one-particle Green's function at $T=0$ is given by

$$
1 / \xi=\frac{4}{U} \int_{1}^{\infty} \frac{\ln \left(y+\sqrt{y^{2}-1}\right)}{\cosh (2 \pi y / U)} \mathrm{d} y .
$$

They obtained formula (2) in a rather heuristic way from an analytic calculation of the finite size $L$ dependence of the Drude weight at $T=0$ [31]

$$
D_{\mathrm{c}}(L)=(-1)^{L / 2+1} L^{1 / 2} D(U) e^{-L / \xi_{\mathrm{c}}(U)} .
$$

Stafford and Millis argued that the correlation length of the one-particle Green's function is identical to the length $\xi_{\mathrm{c}}(U)$ whose computation yielded expression (2). Certainly, in the limiting cases $U \rightarrow 0$ and $U \rightarrow \infty$ formula (2) shows the expected behaviour [31]. However, the status of (2) is that of a conjecture, especially for finite values of $U$, as a proof is still missing for the identity of the finite size scaling length $\xi_{\mathrm{c}}$ and the Green's function correlation length $\xi$. One of the goals of this paper is to confirm formula (2) directly by means of the QTM method and numerical calculations.

In ref. [25], Jüttner et al utilized Shastry's $R$-matrix [32-35] to construct the QTM for the 1D Hubbard model. Therefore, their Bethe ansatz equations are strictly valid only for the coupled spin model, which is obtained from the 1D Hubbard model through the JordanWigner transformation. This transformation poses no problem for the investigation of the bulk properties of the system or the asymptotics of correlation functions such as the spinspin correlations. However, for studying genuinely fermionic correlation functions, especially, the one-particle Green's function, it is necessary to take properly into account its fermionic nature. For this purpose, we employ here the fermionic formulation of the QTM developed by one of the authors [36,37]. (A related fermionic QTM for the lattice spinless fermion model was studied in ref. [27].) Below we sketch the salient points of the fermionic QTM for 
the 1D Hubbard model. First we introduce the fermionic $R$-operator for the 1D Hubbard model [36-38] defined by

$$
\begin{aligned}
\mathcal{R}_{j k}(u, v)= & \mathcal{R}_{j k}^{(\uparrow)}(u-v) \mathcal{R}_{j k}^{(\downarrow)}(u-v)+\frac{\cos (u-v)}{\cos (u+v)} \tanh (h(u)-h(v)) \mathcal{R}_{j k}^{(\uparrow)}(u+v) \\
& \times \mathcal{R}_{j k}^{(\downarrow)}(u+v)\left(2 n_{j \uparrow}-1\right)\left(2 n_{j \downarrow}-1\right), \\
\mathcal{R}_{j k}^{(\sigma)}(u)= & a(u)\left(-n_{j \sigma} n_{k \sigma}+\left(1-n_{j \sigma}\right)\left(1-n_{k \sigma}\right)\right)-b(u)\left(n_{j \sigma}\left(1-n_{k \sigma}\right)+\left(1-n_{j \sigma}\right) n_{k \sigma}\right) \\
& +c(u)\left(c_{j \sigma}^{\dagger} c_{k \sigma}+c_{k \sigma}^{\dagger} c_{j \sigma}\right), \quad(\sigma=\uparrow, \downarrow)
\end{aligned}
$$

where

$$
a(u)=\cos u, \quad b(u)=\sin u, \quad c(u)=1, \quad \frac{\sinh 2 h(u)}{\sin 2 u}=\frac{U}{4} .
$$

Due to the Yang-Baxter equation for the fermionic $R$-operator [38]

$$
\mathcal{R}_{12}\left(u_{1}, u_{2}\right) \mathcal{R}_{13}\left(u_{1}, u_{3}\right) \mathcal{R}_{23}\left(u_{2}, u_{3}\right)=\mathcal{R}_{23}\left(u_{2}, u_{3}\right) \mathcal{R}_{13}\left(u_{1}, u_{3}\right) \mathcal{R}_{12}\left(u_{1}, u_{2}\right)
$$

the row-to-row transfer matrix, $\tau(u)=\operatorname{Str}_{a}\left\{\mathcal{R}_{a L}(u, 0) \cdots \mathcal{R}_{a 1}(u, 0)\right\}$ constitutes a commuting family of operators, $[\tau(u), \tau(v)]=0$. Hence from the logarithmic derivative of the transfer matrix, we obtain the set of commuting local fermionic operators $\left\{I^{(n)}\right\}$,

$$
\tau(u)=\tau(0) \exp \left\{u I^{(1)}+\frac{u^{2}}{2 !} I^{(2)}+\frac{u^{3}}{3 !} I^{(3)}+\cdots\right\},
$$

where $I^{(1)}$ is nothing but the Hamiltonian $\mathcal{H}(1)$. Next, following ref. [36] we introduce the super-transposed $R$-operator $\overline{\mathcal{R}}_{a j}\left(u_{j}, u_{a}\right) \equiv \mathcal{R}_{j a}^{\text {st }_{a}}\left(u_{j}, u_{a}\right)$, as well as a conjugated transfer matrix $\bar{\tau}(u)=\operatorname{Str}_{a}\left\{\overline{\mathcal{R}}_{a L}(0, u) \cdots \overline{\mathcal{R}}_{a 1}(0, u)\right\}$. (See the original reference [36] for the definition of "st" and the explicit form of $\overline{\mathcal{R}}_{a j}\left(u_{j}, u_{a}\right)$.) Since $\bar{\tau}(u)$ is actually expressed as

$$
\bar{\tau}(u)=\operatorname{Str}_{a}\left\{\mathcal{R}_{1 a}(0, u) \cdots \mathcal{R}_{L a}(0, u)\right\},
$$

we can easily deduce

$$
\bar{\tau}(u)=\tau(0)^{-1} \exp \left\{-u \mathcal{H}+O\left(u^{2}\right)\right\} .
$$

Combining (7) and (9) we obtain the fundamental relation

$$
\tau(-u) \bar{\tau}(u)=\exp \left\{-2 u \mathcal{H}+O\left(u^{2}\right)\right\},
$$

which allows us to represent the partition function of the system as

$$
\begin{aligned}
Z & =\operatorname{Tr} \mathrm{e}^{-\beta \mathcal{H}}=\lim _{N \rightarrow \infty} \operatorname{Tr}\left\{\tau\left(-u_{N}\right) \bar{\tau}\left(u_{N}\right)\right\}^{N / 2} \\
& =\lim _{N \rightarrow \infty} \operatorname{Str}\left\{\tau^{\mathrm{QTM}}\left(u_{N}, 0\right)\right\}^{L}, \quad\left(u_{N}=\frac{\beta}{N}\right) .
\end{aligned}
$$

Here $\beta$ is the inverse temperature and $N$ is called the Trotter number. The quantum transfer matrix $\tau^{\mathrm{QTM}}\left(u_{N}, v\right)$ is defined by

$$
\tau^{\mathrm{QTM}}\left(u_{N}, v\right)=\operatorname{Tr}_{j}\left\{\mathcal{R}_{a_{N} j}\left(-u_{N}, v\right) \overline{\mathcal{R}}_{a_{N-1} j}\left(v, u_{N}\right) \cdots \mathcal{R}_{a_{2} j}\left(-u_{N}, v\right) \overline{\mathcal{R}}_{a_{1} j}\left(v, u_{N}\right)\right\} .
$$

From the largest eigenvalue $\Lambda_{0}$ of $\tau^{\mathrm{QTM}}\left(u_{N}, 0\right)$, the free energy per site is obtained in the thermodynamic limit as

$$
\beta f=-\lim _{N \rightarrow \infty} \ln \Lambda_{0}
$$


The correlation lengths $\xi_{i}$ of the (static) correlation functions can be evaluated from the next-leading eigenvalues

$$
\frac{1}{\xi_{i}}=-\Re \ln \left(\Lambda_{i} / \Lambda_{0}\right)
$$

Though the largest eigenvalue $\Lambda_{0}$ is always real and positive, the next leading eigenvalues are not necessarily so. In this case, $\Im \ln \left(\Lambda_{i} / \Lambda_{0}\right)$ is responsible for oscillations of the corresponding correlation function.

The fermionic QTM (12) has been diagonalized [36] by means of the quantum inverse scattering method based on the technique by Ramos and Martins [39, 40]. The eigenstates of the QTM are described by the set of rapidities $\left\{s_{j}, w_{l}\right\},(j=1, \cdots, n, l=1, \cdots, m)$, satisfying the Bethe ansatz equations

$$
\begin{gathered}
\phi\left(s_{j}\right)=-\frac{q_{2}\left(s_{j}-i \gamma\right)}{q_{2}\left(s_{j}+i \gamma\right)}, \quad \frac{q_{2}\left(w_{l}+2 i \gamma\right)}{q_{2}\left(w_{l}-2 i \gamma\right)}=-\frac{q_{1}\left(w_{l}+i \gamma\right)}{q_{1}\left(w_{l}-i \gamma\right)}, \\
q_{1}(s)=\prod_{j}^{n}\left(s-s_{j}\right), \quad q_{2}(s)=\prod_{l}^{m}\left(s-w_{l}\right), \\
\phi(s)=\left(-\frac{\left(1-z_{-} / z(s)\right)\left(1-z_{+} / z(s)\right)}{\left(1+z_{-} / z(s)\right)\left(1+z_{+} / z(s)\right)}\right)^{N / 2},
\end{gathered}
$$

with the following settings

$$
\gamma=\frac{U}{4}, \quad z_{ \pm}=\exp (\alpha)\left\{\tan u_{N}\right\}^{ \pm 1}
$$

and

$$
z(s)=i s\left(1+\sqrt{\left(1-1 / s^{2}\right)}\right), \quad \sinh (\alpha)=-\frac{U}{4} \sin \left(2 u_{N}\right) .
$$

Here, for convenience, we have applied the partial particle-hole transformation to the Bethe ansatz equations derived in [36]. Note that the one-particle Green's function is "invariant" under the partial particle-hole transformation.

The corresponding eigenvalue of the fermionic QTM, $\tau^{\mathrm{QTM}}\left(u_{N}, 0\right)$ is given by

$$
\Lambda=c\left(u_{N}\right) \prod_{j=1}^{n} z_{j},
$$

where $z_{j} \equiv z\left(s_{j}\right)$. We omit here the precise form of the prefactor $c\left(u_{N}\right)$, which is in common for all eigenstates and is not necessary for the evaluation of the correlation lengths.

We like to emphasize that the fermionic statistics is properly incorporated in the Bethe ansatz equations (15) and the eigenvalue formula (19). For example, we have found that the dominant eigenvalue contributing to the correlation length for $\left\langle c_{k \sigma}^{\dagger} c_{j \sigma}\right\rangle$ is the largest eigenvalue in the sector $[n=N-1, m=N / 2-1]$. In Fig. 1 (b), we plot an example of the distribution pattern of the rapidities $z_{j}$ for the dominant eigenvalue $\Lambda_{\mathrm{G}}$. As all rapidities $z_{j}$ turn out to be purely imaginary, we plot them in a 90-degree rotated frame, i.e., the horizontal line is the imaginary axis. Compared with $z_{j}$ for the largest eigenvalue $\Lambda_{0}$ (see Fig. 1 (a)), we can roughly say that one of the $z_{j}$ closest to the origin is removed. Note that the complex conjugate of $\Lambda_{\mathrm{G}}$ is also the largest eigenvalue of the sector $[n=N-1, m=N / 2-1]$ and the corresponding rapidities $z_{j}$ are given by the reflection of those for $\Lambda_{\mathrm{G}}$ with respect to the real axis (the vertical 

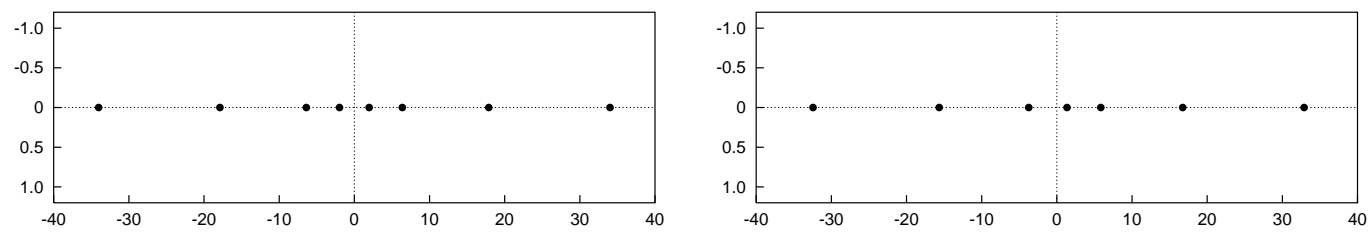

Fig. 1 - (a) The rapidities $z_{j}$ on the imaginary axis (horizontal line) for the largest eigenvalue $\Lambda_{0}$ in the sector $N=8, n=8, m=4, U=8, \beta=0.8$. (b) The rapidities $z_{j}$ on the imaginary axis (horizontal line) for the largest eigenvalue $\Lambda_{\mathrm{G}}$ in the sector $N=8, n=7, m=3, U=8, \beta=0.8$

line in Fig. 1). From the expression (19) and the distribution pattern of $z_{j}$ in Fig. 1 (b), we find $\Lambda_{\mathrm{G}}$ to be purely imaginary. This is consistent with the fact that the one-particle Green's function should exhibit $k_{\mathrm{F}}=\pi / 2$ oscillations at half-filling. We have numerically solved the Bethe ansatz equations (15) for increasing Trotter numbers $N$ and calculated the correlation length $\xi$ from the ratios of the eigenvalues $\Lambda_{\mathrm{G}}$ and $\Lambda_{0}$. The result is plotted in Fig. 2. The largest Trotter number we took is $N=10240$ down to temperatures as low as $T=0.001$. For such low temperatures, we have extrapolated our data for $N=6144,8192,10240$ to estimate the limiting behaviour for $N \rightarrow \infty$. The error of the extrapolated results is less than $0.5 \%$. For moderate temperatures $(T>0.01)$, it was sufficient to use Trotter numbers just about $N=4096$ to get very accurate data.

From Fig. 2, we can clearly observe that the correlation lengths remain finite as $T \rightarrow 0$. The extrapolation of our data to $T \rightarrow 0$ is compared with (2) in Fig. 3 (a). To perform the extrapolation, we assumed the low temperature behaviour of the correlation length in the form $\xi=\xi_{T=0} \times \exp (-\gamma T)$ and estimated $\xi_{T=0}$ from our numerical data at low temperatures. Obviously our extrapolations coincide with formula (2) almost perfectly. Therefore we conclude Stafford and Millis' conjecture is correct for any value of $U$.

Having confirmed that formula (2) gives the correct correlation length $\xi$ at $T=0$, we now look at its dependence on the gap $\Delta$, see Fig. 3 (b). Here, we used the well known gap formula

$$
\Delta=\frac{16}{U} \int_{1}^{\infty} \frac{\sqrt{y^{2}-1}}{\sinh (2 \pi y / U)} \mathrm{d} y
$$

Usually the correlation length of a gapped system is believed to be simply inversely propor-
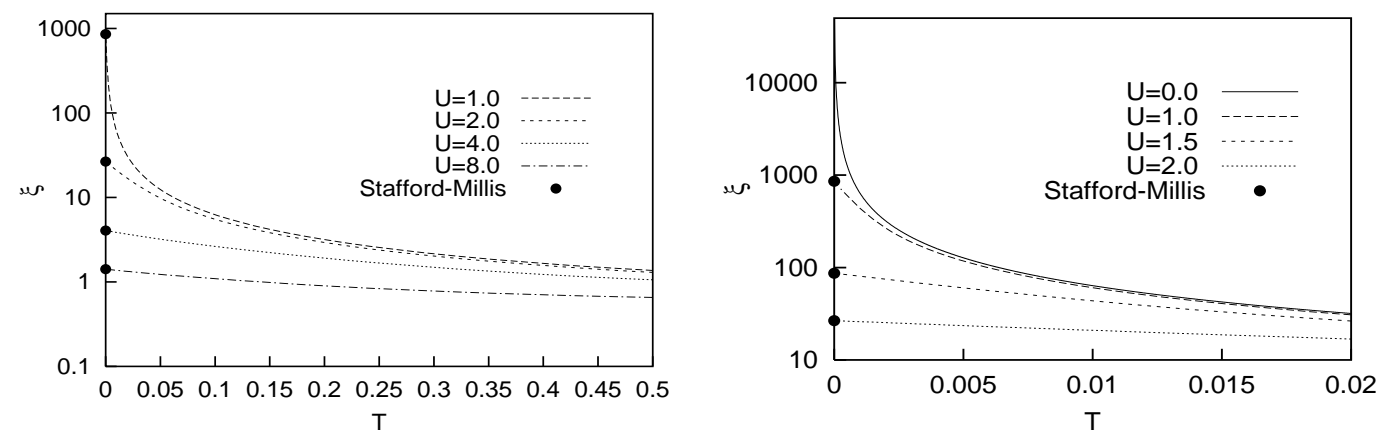

Fig. 2 - (a) Temperature dependence of the correlation lengths for one-particle Green's function for the half-filled Hubbard model. (b) Similar to (a) at lower temperatures 
tional to the magnitude of the gap. Equations (2) and (20) indeed give $\xi^{-1} \propto \Delta$ for small $U$, however $\xi^{-1} \propto \ln \Delta(\sim \ln U)$ for large $U$ [31]. More precisely, we have

$$
\begin{array}{lll}
\xi^{-1} & \sim \Delta / 4 & (U \rightarrow 0), \\
\xi^{-1} & \sim \ln (\Delta / a) & (U \rightarrow \infty),
\end{array}
$$

where $a=\left[\Gamma\left(\frac{1}{4}\right) / \sqrt{2 \pi}\right]^{4} \simeq 4.37688$ (see ref. [31]). Thus the gap dependence of the correlation length does not follow a simple rule. For a discussion of this issue we like to refer the reader to ref. [41].

Finally, for increasing temperature $T$, the correlation length $\xi$ is getting closer to the non-interacting free-fermion $(U=0)$ value, namely $\xi_{U=0}=1 / \ln \left(\pi T / 2+\sqrt{(\pi T / 2)^{2}+1}\right)$, irrespective of the value of $U$. Actually, for small $U(\sim 1)$, the correlation length $\xi$ takes almost the same value as $\xi_{U=0}$ even at rather low temperatures $(T \sim 0.01)$

In this paper we have presented a numerical treatment of the one-particle correlation length $\xi(T)$ for finite Trotter number $N$. In a future publication, we will derive the NLIE for the corresponding eigenvalue $\Lambda_{\mathrm{G}}$ in the limit $N \rightarrow \infty$. This will allow us to obtain analytic results for the $T \rightarrow 0$ limit and to prove formula (2) analytically.

After completing this manuscript we found a derivation of the zero temperature correlation length (2) on the basis of the standard row-to-row transfer matrix of the Hubbard model and the one-particle energy-momentum excitation following the method described in [41]. Details of this calculation will be published elsewhere.
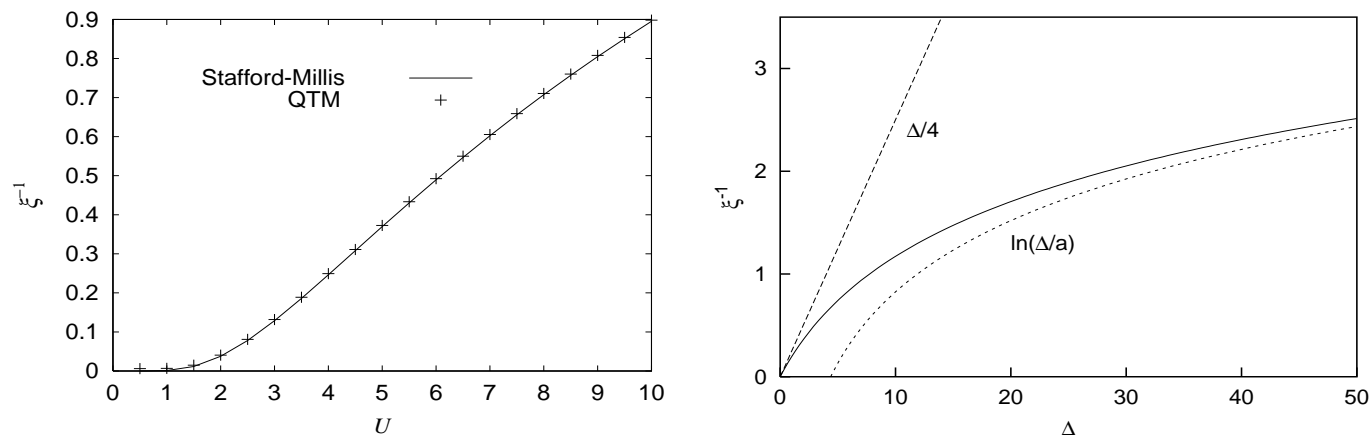

Fig. 3 - (a) Comparison of data obtained from an extrapolation of the finite temperature correlation length to $T=0$ with Stafford and Millis' conjecture. (b) The dependence of the $T=0$ correlation length $\xi$ on the charge gap $\Delta$. The dotted lines correspond to the asymptotics (21) and (22).

$$
* * *
$$

The authors are grateful to M. Wadati, M. Takahashi, M. Inoue, K. Sakai and K. Saito for valuable discussions and continuous encouragements. They also thank the Supercomputer Center, Institute for Solid State Physics, University of Tokyo for the use of the facilities.

\section{REFERENCES}

[1] E. H. Lieb and F. Y. Wu : Phys. Rev. Lett. 20 (1968) 1445; Preprint 2002, cond-mat/0207529 
[2] V. E. Korepin and F. H. L. Essler : "Exactly Solvable Models of Strongly Correlated Electrons", World Scientific, (1993)

[3] M. Takahashi "Thermodynamics of One-Dimensional Solvable Models", Cambridge University Press, (1999).

[4] T. Deguchi, F. Essler, F. Göhmann, A. Klümper, V. E. Korepin and K. Kusakabe : Phys. Rep. 331 (2000) 197.

[5] M. Takahashi : Prog. Theor. Phys. 47 (1972) 69.

[6] M. Takahashi : Prog. Theor. Phys. 52 (1974) 103.

[7] N. Kawakami, T. Usuki and A. Okiji : Phys. Lett. A 37 (1989) 287.

[8] T. Usuki, N. Kawakami and A. Okiji : J. Phys. Soc. Jpn. 59 (1990) 1357.

[9] M. Takahashi and M. Shiroishi : Phys. Rev. B 65 (2002) 165104.

[10] M. Suzuki and M. Inoue : Prog. Theor. Phys. 78 (1987) 787.

[11] T. Koma : Prog. Theor. Phys. 78 (1987) 1213; 81 (1989) 783.

[12] M. Inoue and M. Suzuki : Prog. Theor. Phys. 79 (1988) 645.

[13] M. Wadati and Y. Akutsu : Prog. Theor. Phys. 94 (1988) 1.

[14] T. Koma : Prog. Theor. Phys. 83 (1990) 655.

[15] M. Yamada : J. Phys. Soc. Jpn. 59 (1990) 848.

[16] J. Suzuki, Y. Akutsu and M. Wadati : J. Phys. Soc. Jpn. 59 (1990) 2667.

[17] M. Takahashi : Phys. Rev. B 43 (1991) 5788; 44 (1991) 12382.

[18] K. Tsunetsugu : J. Phys. Soc. Jpn. 60 (1991) 1460.

[19] J. Suzuki, T. Nagao and M. Wadati : Int. J. Mod. Phys. B 6 (1992) 1119.

[20] A. Klümper : Ann. Phys. 1 (1992) 540; Z. Phys. B 91 (1993) 507.

[21] H. Mizuta, T. Nagao and M. Wadati : J. Phys. Soc. Jpn. 63 (1994) 3951.

[22] A. Klümper and R. Z. Bariev : Nucl. Phys. B 458 (1996) 623.

[23] H. Mizuta and M. Wadati : J. Phys. Soc. Jpn. 66 (1997) 988, ibid 3741.

[24] G. Jüttner, A. Klümper and J. Suzuki : Nucl. Phys. B 512 (1998) 581.

[25] G. Jüttner, A. Klümper and J. Suzuki : Nucl. Phys. B 522 (1998) 471.

[26] A. Kuniba, K. Sakai and J. Suzuki : Nucl. Phys. B 525 (1998) 597.

[27] K. Sakai, M. Shiroishi, J. Suzuki and Y. Umeno : Phys. Rev. B60 (1999) 5186.

[28] K. Sakai : J. Phys. Soc. Jpn. 68 (1999) 1789.

[29] A. Klümper and J. R. Martinez, C. Scheeren, M. Shiroishi : J. Stat. Phys. 102 (2001) 937.

[30] K. Sakai and A. Klümper : J. Phys. A: Math. Gen. 39 (2001) 8015.

[31] C. A. Stafford and A. J. Millis : Phys. Rev. B 48 (1993) 1409.

[32] B. S. Shastry : Phys. Rev. Lett. 56 (1986) 1529, ibid 2453.

[33] E. Olmedilla, M. Wadati and Y. Akutsu : J. Phys. Soc. Jpn. 56 (1987) 1340.

[34] B. S. Shastry : J. Stat. Phys. 50 (1988) 57.

[35] M. Shiroishi and M. Wadati : J. Phys. Soc. Jpn. 64 (1995) 57, ibid 2795, 4598.

[36] Y. Umeno : J. Phys. Soc. Jpn. 70 (2001) 2531.

[37] Y. Umeno : Doctor thesis, University of Tokyo (2002).

[38] Y. Umeno, M. Shiroishi and M. Wadati : J. Phys. Soc. Jpn. 67 (1998) 2242.

[39] P. B. Ramos and M. J. Martins : J. Phys. A: Math. Gen. 30 (1997) L195.

[40] M. J. Martins and P. B. Ramos : Nucl. Phys. B522 (1998) 413.

[41] K. Okunishi, Y. Akutsu, N. Akutsu, T. Yamamoto : Phys. Rev. B 64 (2001) 104432. 